\title{
How Digital Nudges Affect Consideration Set Size and Perceived Cognitive Effort in Idea Convergence of Open Innovation Contests
}

\author{
Ivan Boskovic-Pavkovic \\ Convergent Media Group, \\ Croatia \\ iv8boskovic@gmail.com
}

\author{
Isabella Seeber \\ University of Innsbruck, \\ Austria \\ isabella.seeber@uibk.ac.at
}

\author{
Ronald Maier \\ University of Innsbruck, \\ Austria \\ ronald.maier@uibk.ac.at
}

\begin{abstract}
Open innovation initiatives are useful to acquire many ideas, but often face problems when it comes to selecting the best ideas. Idea convergence has been suggested as a first step in idea selection to filter those ideas that are worthy of further consideration. Digital nudges - digital interventions that aim at altering human behavior in a predictable way - could support convergence. However, their effects are largely unknown. This study explores how two digital nudges, selection strategy (inclusion/exclusion) and idea subset similarity (similar/random), affect the convergence outcomes consideration set size and perceived cognitive effort. We conducted a laboratory experiment with 88 students and found that guiding individuals towards an inclusion strategy results in smaller consideration sets and higher perceived cognitive effort. Moreover, presenting individuals with subsets of similar ideas resulted in smaller consideration sets. These insights are relevant for the design and use of digital nudges for convergence in open innovation environments.
\end{abstract}

\section{Introduction}

Organizations increasingly employ crowds to enhance their innovation processes, which has been termed open innovation (OI) [1]. Innovation contests are one form of OI initiatives, in which a crowd generates hundreds or thousands of ideas from which only a few will be selected as the best ideas [2]. Research has established extensive insights and knowledge into the idea creation process, but has not yet developed sufficient understanding to explain the idiosyncrasies of idea selection processes [3].

\footnotetext{
1 https://blog.hypeinnovation.com/product/pairwise-evaluationinnovation-platform-hype-enterprise
}

During idea selection, a small team usually first converges on the 10 to 40 most promising ideas within a few days or weeks followed by a jury with high domain expertise that determines the winning idea(s) [4], [5]. Organizations struggle with adopting appropriate convergence procedures when facing large pools of generated ideas [5]. More and more organizations attempt to outsource this process to the crowd [2]. However, individual crowd raters are limited in their ability to select the best ideas [3]. They experience high levels of cognitive load [6] and are influenced by cognitive biases [7], because of the often number of ideas they need to process [4] and limited knowledge of how the idea came about [3], [8].

Idea selection is not new, but developments in technology provide new capabilities that continuously alter the way how individuals make their choices [9]. For example, the innovation platform Hype ${ }^{1}$ incorporates amongst others a pairwise comparison feature for ideas so that raters can more easily select those ideas that perform well according to specific quality criteria.

The design of meaningful features to support convergence also sparked the interest of researchers who investigated e.g., the design of adequate rating scales [10], meaningful presentation modes of ideas [7] or targeted digital facilitation intervention [11].

We propose that digital nudges, digital interventions that alter "people's behavior in a predictable way" [12], could help raters to experience lower perceived cognitive effort during convergence and achieve higher reduction rates (smaller consideration set sizes). Digital nudging aims at explaining how user-interface design elements affect individual's information processing and choices [13]. It is crucial to identify influential digital nudges that are able to alter human behavior to such extent that they can significantly drive convergence outcomes. While past research has identified a number 
of digital nudges, we still lack conceptual understanding of how those digital nudges affect behavior and outcomes [13], specifically in the application domain of idea selection as part of open innovation initiatives.

This paper aims at addressing this gap, by investigating the effects of two digital nudges on convergence outcomes. We draw on an online laboratory experiment involving 88 participants to empirically test the effects of selection strategy and idea similarity on consideration set size and perceived cognitive effort.

\section{Background}

\subsection{Digital nudging}

Nudges describe small objects placed into an environment to steer people in particular directions. This could be the placement of nutritious food into the middle of a food court or having a painted fly on a men's urinal [14]. They are interventions into human behavior and are more than the mere provision of factual information or rational persuasion [12]. Hansen defines a nudge as [12]:

" a function of (I) any attempt at influencing people's judgment, choice or behavior in a predictable way, that is (1) made possible because of cognitive boundaries, biases, routines and habits in individual and social decision-making posing barriers for people to perform rationally in their own declared self-interests, and which (2) works by making use of those boundaries, biases, routines, and habits as integral parts of such attempts."

Multiple types of nudges have been identified, such as default rules, active choosing, increase in ease and convenience, simplification, warnings, or reminders and their effects have been studied in domains such as policy making [15]. Recently, also the term digital nudge emerged to describe nudges in the form of user-interface design elements that exist in online choice environments [13], in our case an idea convergence platform. These design elements alter information processing in order to affect our judgments and choices [16].

\subsection{Dual processing and convergence outcomes}

Dual processing theory suggests that there are two systems at work when humans judge and engage in decision-making [17].

System 1 processing is unconscious, implicit, automatic, requires low effort, and is rapid [18]. In contrast, system 2 processing is conscious, explicit, controlled requires high effort and is slow [18], but should lead to highly accurate choices [19]. System 1 processing is prone to cognitive biases, which may result in imperfect decisions [17], [20]. Such biases are systematic errors in our decision making [20] and might therefore be detrimental to successful idea convergence. Decision makers might not process all ideas in depth to come to an informed decision and hence are more likely to miss good ideas.

Consequently, perceived cognitive effort might be a promising convergence outcome to be investigated, because it indicates to what extent decision makers engaged in effortful thinking. In addition, the success of overall idea selection might depend on adequate consideration set sizes after convergence. If consideration sets are too large, more resources, i.e. experts' time, are required to make a final selection and experts might be overloaded thus hampering the decision outcome. If consideration sets are too small, the variety of ideas might appear too small and thus affect experts' confidence in making an informed decision to determine the winner idea(s). This suggests that successful convergence should also consider consideration set size as an indicator of performance.

\section{Hypotheses}

\subsection{Selection strategy}

Prompting raters towards eliminating bad ideas (lemons) showed to achieve higher evaluation accuracy than prompting them towards selecting good ideas (stars) [21]. The task prompt to choose good ideas or eliminate bad ideas can be connected to theory on inclusion and exclusion strategies [22], [23]. With inclusion, a decision maker decides whether an "option should be seriously considered for a final choice" [22, p. 1194]. With exclusion, the decision maker decides whether an "option should be dropped from further consideration" [22, p. 1194].

Independent of the task type, decision makers that adopt an exclusion strategy are likely to end up with larger consideration set sizes [22], [23]. Rietzschel et al. [24] confirmed that the hypothesis of larger consideration set sizes for exclusion also holds true for the selection of creative ideas. In their experiment, participants self-generated the ideas that they later converged on. It remains empirically unexplored, if theory of larger consideration set sizes under exclusion holds when decision makers converge on ideas that are not their own as is the case for idea convergence in open innovation initiatives.

In addition, information-processing behavior differs for individuals that follow an inclusion compared to an exclusion strategy. Past research showed that decision 
makers adopting an inclusion strategy examine more attributes of an alternative (breadth) and spend more time on an alternative (depth). This observation was particularly pronounced for individuals with high need for cognition [23]. In-depth processing might be even higher when decision makers are prompted to select ideas that are not their own, because they have limited information about the idea [25]. Hence, the basic mechanism behind such a digital nudge "selection strategy" is to alter the depth and breadth of information processing of alternatives and their attributes.

In an online environment, a task prompt in connection with a check box or toggle switch provides the necessary means to nudge decision makers either into including or excluding ideas. This is a nudge of type active choosing [15], which lets decision makers make a conscious decision. This means in any case, the decision maker needs to act to choose an idea (inclusion condition) or drop an idea (exclusion condition). Thus,

H1: Decision makers tasked to reduce ideas by inclusion will have a smaller consideration set than decision makers tasked to reduce ideas by exclusion.

H2: Decision makers tasked to reduce ideas by inclusion will have higher perceived cognitive effort than decision makers tasked to reduce ideas by exclusion.

\subsection{Idea subset similarity}

Many idea selection initiatives struggle with a high number of similar and duplicate ideas submitted to a contest [26]. The extent of idea similarity can provide indication about the quality of an idea but research findings are heterogeneous whether similarity of ideas is a good or bad indicator of idea quality e.g., [27]-[29]. Even more under-researched is our understanding of how similarity among ideas affects the decision making processes. Field research indicates that categorizing ideas makes the reduction of ideas easier [5]. A reason for this provides extant literature in consumer research, which showed that a lack of categorization may contribute to choice overload [30]. Choice overload sets in when a decision maker is faced with a complex decision, which exceeds his or her individual cognitive resources [31]. Hence, when being confronted with uncategorized alternatives, decision makers find it harder to navigate the choice set [30] because the extracted features are not comparable. When similar alternatives are presented and therefore overlaps in the alternatives' features exist [32] the choice should become easier. The digital nudge "idea subset similarity" is of type increase in ease and convenience, which is implemented as a similarity feature (see
Section 4) and aims at reducing perceived difficulty [15].

The basic mechanism behind this nudge is that it makes it easier to compare features of alternatives when similar ideas are presented and can be analyzed at once. When features of alternatives are not aligned (dissimilar idea sets), decision makers show higher search costs [33], which might indicate higher perceived cognitive effort. When the demand on perceived cognitive effort increases to such an extent that decision makers experience choice overload, they should be less likely to make a choice at all (choice deferral) [34]. When applying this line of argument to the context of idea convergence, this suggests that choosing from a set of dissimilar ideas should result in larger consideration set sizes, because decision makers would defer their choices and more perceived cognitive effort is required due to increased search cost. In case of choosing from similar ideas, decision makers should end up with smaller consideration set sizes and experience lower perceived cognitive effort, because it is easier for them to compare the features inherent to the ideas and make a choice. Thus,

H3: Decision makers presented with subsets defined by idea similarity will have a smaller consideration set than decision makers presented with random subsets.

H4: Decision makers presented with subsets defined by idea similarity will perceive lower perceived cognitive effort than decision makers presented with random subsets.

\section{Method}

We conducted a $2 \times 2$ between-subjects laboratory experiment to explore the associations between our two manipulated convergence mechanisms selection strategy and idea similarity and the two dependent variables consideration set size and perceived cognitive effort. In the following, we describe the experimental design and the methods we used to analyze the data collected.

\subsection{Manipulation}

We implemented the two digital nudges (1) selection strategy with the two options of inclusion and exclusion (active choosing) and (2) idea similarity with the two options similarity-based and random decomposition of ideas into subsets (increase in ease and convenience) in an online convergence platform using HTML5, AJAX, jQuery, Javascript, mySQL and PHP. This system allowed us to present participants with four treatments 
and to collect data on their choices, demographic data and participants' perceptions on the decision process and outcome.

Selection strategy. The system provides the participants with a task explanation that states that they can either "choose" or "drop" ideas. The task prompt conveyed that experts will consider the lists of ideas selected by participants. This aimed at framing the experimental task as idea convergence and not winner determination. Furthermore, the ideas have a checkbox next to them which, when selected, displays either the " $\checkmark$ " symbol for the decision making variable "choose" (Figure 1) or the symbol " $\boldsymbol{x}$ " for the "drop" variable (Figure 2). Hence, in the inclusion treatment subjects had to actively choose the " $\checkmark$ " box, whereas subjects in the exclusion treatment had to actively choose the " $x$ " box. We refrained from using red and green colors to not adversely affect subjects that are red-green colorblind.

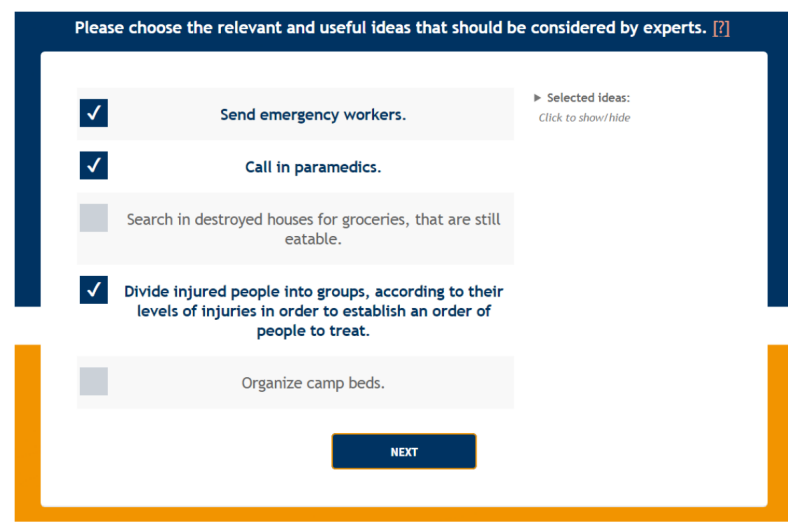

Figure 1: Selection strategy: inclusion

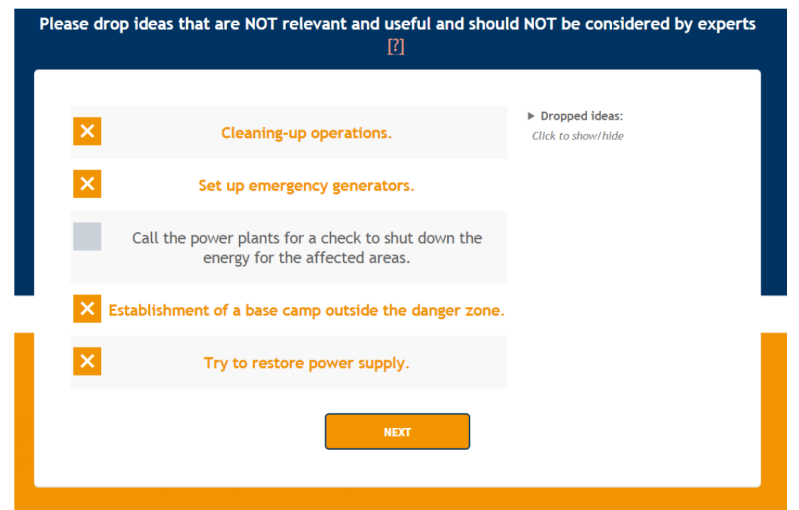

Figure 2: Selection strategy: exclusion

Idea subset similarity. Our platform divides the initial set of generated ideas into subsets of five ideas that are considered in separate rounds. This division into subsets of five ideas, which represents a small choice set [34], was held constant for all treatments. This should facilitate idea convergence as it helps guide the attention to a few ideas at a time [11]. Still, given the context of the study, individuals are likely to experience cognitive overload even with small choice sets because the decision task is difficult, individual's preference is uncertain, and the overall choice set is complex [34]. We implemented the digital nudge "idea subset similarity" so that the platform presents either random or similaritybased subsets of ideas, but no further indication, e.g., category description, was provided.

For random decomposition of idea subsets, our convergence platform calls a subset of five ideas out of the total set of 75 ideas from a MySQL database system at a time with a simple random function that the database management system offers. The system repeats this drawing of five ideas until all ideas from the initial idea pool have been shown to participants exactly once.

For decomposition of similar idea subsets, the platform selects the five most similar ideas out of the idea pool, removes them from the pool and repeats the drawing until all ideas are presented exactly once. We measure the similarity of ideas with an algorithm that determines and weighs similarity between ideas. While generally there is a plethora of text analysis techniques, many focus on comparing two long text fragments or a short to a long one [35], [36]. As our idea pool contains short sentences, it is difficult to apply those machine learning algorithms to determine similarity. Therefore, we devised a custom algorithm.

The algorithm first performs some text preprocessing, i.e. removal of stop words. The algorithm then acquires the synonyms of all remaining words from the WordNet ontology database ${ }^{2}$ that offers English nouns, verbs, adjectives and adverbs grouped into sets of cognitive synonyms and interlinked by means of conceptual-semantic and lexical relations, resulting in a network of meaningfully related words and concepts. We employ the WordNet API to get the synonyms for each word of an idea using a threshold of 30 for the semantic distance calculated by WordNet, as this threshold marks a noticeable decrease in the similarity between the original word and synonyms beyond this distance. Every idea is therefore enriched by its synonyms.

The algorithm selects an enriched idea as reference idea and compares it to every other idea in the set, thus calculating a similarity metric of the number of matched words between two ideas, both original words and synonyms. The algorithm creates a subset of the reference idea plus the four ideas with the highest values on this similarity metric and records the subset's total

\footnotetext{
2 https://wordnet.princeton.edu
} 
similarity score. The algorithm then removes these five ideas from the idea pool and repeats this drawing process until all ideas have been shown to participants exactly once.

We found this algorithm to yield the highest total similarity score (2,737 matches of words) out of a series of options we experimented with.

\subsection{Subjects}

We recruited 93 bachelor students from the introductory course to Information Systems at a European university. We deem students as appropriate representatives of crowd workers, who are predominantly young, hold a bachelor's degree and have a rather low income [37]. Their participation was voluntary and they received course credit for their participation. While the experiment was in English, the course language was German. Due to the fact that English proficiency is high throughout the university and no problems related to language were raised during or after the experiment, we do not expect that there existed a language barrier that could have affected the results of the experiment. The participants' average age was 21.5 years.

\subsection{Measures}

We measured the two dependent variables consideration set size plus perceived cognitive effort and controlled for gender, domain expertise, and need for cognition.

Consideration set size describes the number of ideas retained after the convergence task [24]. For subjects in the inclusion treatment, we counted the number of ideas they selected into their consideration set. For subjects in the exclusion treatment, we counted the number of ideas they did not exclude and therefore remained in their consideration set.

Perceived cognitive effort refers to the degree of engagement with a demanding task [38]. We adopted three items [39], [40] and adapted the items to correspond to the study's context: "The task of selecting ideas took too much time", "Selecting ideas required too much effort", and "Selecting ideas was too complex".

Controls. We controlled for gender, domain expertise, and need for cognition. Gender was part of the demographic data we collected and found to be strongly correlated with consideration set size and hence taken up as control in our further data analysis. Domain expertise and need for cognition were included, because they have been identified as relevant factors for choice overload [34] and therefore the perception of cognitive effort. We self-developed the construct of domain expertise by formulating the five items: "I have expertise in the topic of emergency response.", "I have experience with emergency response.", "I am very competent in the topic of emergency response.", "I am not familiar with the topic of emergency response. (r)", and "Emergency response interests me." We adopted five items from [41] to measure need for cognition. The scale assesses to what extent individuals engage and enjoy thinking with the items "I don't like to have to do a lot of thinking.(r)", "I try to avoid situations that require thinking in-depth about something.(r)", "I prefer complex problems over simple problems.", "I prefer to do something that challenges my thinking abilities rather than something that requires little thought.", and "Thinking hard and for a long time about something gives me little satisfaction. (r)".

\subsection{Procedure and task}

The experiment consisted of the three phases (1) task description and consent, (2) convergence task and (3) post-task survey.

Students were (1) informed about our experiment and that participation is voluntary, anonymous and incentivized by course credit. They signed a consent form if they volunteered to participate. We also informed participants that there is no time limit for completing the experiment and that one of the authors was available to answer any questions they might have or help with technical difficulties that they might experience.

Concerning the (2) convergence task, emails with a link to the web page which hosted the convergence platform were sent before class to each student taking the course. We randomly assigned students into one of four treatment groups resulting from a $2 \times 2$ factorial design. In each treatment group, participants were tasked to converge on a total of 75 ideas, decomposed into 15 subsets of five ideas presented per page. The ideas suggested help measures to tackle the problems of a fictional town "Norvos" getting hit by extreme flooding which was based on real events. The ideas had been generated in a previous crowdsourcing initiative. Our participants' task was, based on their treatment group, to choose (drop) the help measures that they deemed relevant and useful (neither relevant, nor useful) and that should (should not) be further considered by a team of emergency response experts. Participants were informed that they could select any number of ideas between zero and five per page.

We finally asked participants to complete a (3) posttask survey with which we collected data on perceived 
cognitive effort, need for cognition, domain expertise, and demographics.

\section{Results}

Manipulation check and outliers. To check whether a subject correctly perceived the selection strategy, we included the item "What decision making choice were you presented with? [choose idea or drop idea]. We had to exclude four subjects who were tasked to drop ideas but answered that their task was to choose ideas. To check if subjects correctly perceived idea set similarity, we included the item "To what extent did you perceive ideas in sets of 5 to be similar?" and measured it on a 5-point Likert scale. We performed an ANOVA to assess whether subjects perceived the idea sets in the similarity treatment as more similar than in the random treatment. Results indicate a non-significant effect $(\mathrm{F}(1,86)=0.049, \mathrm{p}>$ $0.05)$. Our participants perceived the ideas as somewhat similar in both the similarity treatment $(M=2.10$; $\mathrm{SD}=0.69)$ and the random treatment $(\mathrm{M}=2.07 ; \mathrm{SD}=0.57)$. Moreover, we dismissed data of one student as an outlier, because he finished the task within 59 seconds, whereas the average time spent in his treatment group was 13:08 minutes. Across all treatment groups participants needed 13:54 minutes on average to complete the experiment. The final dataset contained 88 subjects of which were 44 female and 44 male students, which were unequally distributed between treatments (Table 1).

Reliability and validity. We performed reliability analysis and exploratory factor analysis for domain expertise, perceived need for cognition and cognitive effort in an iterative manner to establish reliability and validity of our perceptual constructs. Cronbach's Alphas for perceived cognitive effort and need for cognition was below the recommended threshold of 0.7 [42]. Reliability of perceived cognitive effort could have been improved by dropping the third item, but we decided against that in order to follow the recommendation to measure perceptual constructs with at least three items [43]. Also the Exploratory Factor Analysis (EFA with Promax rotation) revealed in our first attempt a four-factor instead of a three-factor solution. Two items of need for cognition and one item for domain knowledge loaded poorly on their latent factor. Therefore, we dropped those items and repeated reliability and validity analyses. After revision, we deemed Cronbach's Alphas acceptable for perceived cognitive effort $(\alpha=0.656)$, need for cognition $(\alpha=$ $0.680)$, and domain knowledge $(\alpha=0.921)$. Also convergent validity was deemed satisfactory with factor loadings $>0.5$ and Measures of Sampling Adequacy $(\mathrm{MSA})>0.5$. Also discriminant validity was deemed satisfactory as the pattern matrix suggested a threefactor solution [43]. We then used an averaged scale for domain expertise and created a binary variable for need for cognition using median split to interpret low and high need for cognition individuals.

Controls. We ran ANOVAs on gender, domain expertise, and need for cognition. There were no significant differences between treatments for each of the control variables $\left(\mathrm{F}_{\text {gender }}=1.028, \mathrm{~F}_{\text {domain expertise }}=\right.$ $0.185, \mathrm{~F}_{\text {need_for_cognition }}=1.920$ ).

Tests of assumptions for MANCOVA. We added gender, domain expertise, and need for cognition as covariates and performed a MANCOVA specifying selection strategy (choose, drop) and idea similarity (similar, random) as independent variables and consideration set size and perceived cognitive effort as dependent variables. The descriptive statistics are given in Table 1. Concerning the assumption of equality of variance-covariance matrices, we assessed the Box's M test using the recommended conservative significance level of 0.01 [43]. The test statistic indicates that there exist no significant differences between treatment groups $(\mathrm{p}>0.01)$ and therefore the assumption is not violated. Concerning the assumption of multivariate normal distribution, which cannot be directly tested in SPSS, we fell back to the common procedure to assess normal distribution for each dependent variable per treatment group with the Shapiro-Wilk test [43]. All normality tests showed insignificant values $(p>0.05)$ indicating that the data does not significantly deviate from a normal distribution. We also drew on scatterplots, which visually supported this statistical result.

Table 1: Descriptive statistics of treatment groups

\begin{tabular}{lcccc}
\hline & $\begin{array}{l}\text { Choose } \\
\text { random }\end{array}$ & $\begin{array}{l}\text { Choose } \\
\text { similar }\end{array}$ & $\begin{array}{l}\text { Drop / } \\
\text { random }\end{array}$ & $\begin{array}{l}\text { Drop / } \\
\text { similar }\end{array}$ \\
\hline $\begin{array}{l}\text { \# of } \\
\text { subjects }\end{array}$ & 20 & 25 & 22 & 21 \\
\hline \# of & 39.25 & 33.80 & 62.14 & 59.76 \\
selected & $(9.13)$ & $(10.53)$ & $(5.00)$ & $(7.18)$ \\
ideas & & & & \\
\hline $\begin{array}{l}\text { Perceived } \\
\text { cognitive }\end{array}$ & 3.63 & 3.43 & 3.12 & 3.11 \\
effort & $(0.59)$ & $(0.61)$ & $(0.85)$ & $(0.64)$ \\
\hline Gender & $10 \mathrm{~m} /$ & $9 \mathrm{~m} /$ & $13 \mathrm{~m} /$ & $12 \mathrm{~m} /$ \\
& $10 \mathrm{f}$ & $16 \mathrm{f}$ & $9 \mathrm{f}$ & $9 \mathrm{f}$ \\
\hline $\begin{array}{l}\text { Domain } \\
\text { expertise }\end{array}$ & 4.03 & 4.09 & 4.18 & 3.98 \\
\hline $\begin{array}{l}\text { Need for } \\
\text { cognition }\end{array}$ & $1.9 .80)$ & $(1.01)$ & $(0.91)$ & $(1.08)$ \\
\hline
\end{tabular}


Hypotheses testing. The multivariate test statistic shows a significant main effect for selection strategy (Pillai's trace $=0.735, \mathrm{~F}(2,80)=111.168, \mathrm{p}<0.001)$, idea similarity (Pillai's trace $=0.079, \mathrm{~F}(2,80)=3.427, \mathrm{p}$ $=0.037$ ), gender (Pillai's trace $=0.095, \mathrm{~F}(2,80)=4.215$, $\mathrm{p}=0.018$ ), and need for cognition (Pillai's trace $=0.097$, $\mathrm{F}(2,80)=4.308, \mathrm{p}=0.017)$. There was no significant main effect for the covariate domain expertise (Pillai's trace $=0.050, \mathrm{~F}(2,80)=2.119, \mathrm{p}>0.05)$. There was also no significant multivariate interaction effect (Pillai's trace $=0.009, \mathrm{~F}(2,80)=0.688, \mathrm{p}>0.05)$. Hypotheses testing proceeded with interpreting the univariate test statistics, which are summarized in Table 2.

H1 suggested that consideration sets would be smaller and $\mathrm{H} 2$ suggested that perceived cognitive effort would be higher for decision makers who chose ideas (inclusion) than for decision makers who dropped ideas (exclusion). The univariate test statistics show significant differences for consideration set size (F (1, $81)=208.825, p<0.001$, partial $\left.\eta^{2}=0.721\right)$ and perceived cognitive effort $(\mathrm{F}(1,81)=5.268, \mathrm{p}=0.024$, partial $\left.\eta^{2}=0.061\right)$. Decision makers who chose ideas had on average 36.22 out of 75 ideas in their consideration sets in contrast to 60.98 out of 75 ideas for decision makers who dropped ideas. Hence, directing decision makers towards choosing ideas resulted in smaller consideration sets, which supports H1. Moreover, decision makers perceived on average higher cognitive effort when choosing ideas $(\mathrm{M}=3.52)$ than when dropping ideas $(\mathrm{M}=3.12)$. Therefore, also $\mathrm{H} 2$ is supported.

$\mathrm{H} 3$ and $\mathrm{H} 4$ were concerned with the effects of idea similarity and suggested that consideration sets would be smaller and perceived cognitive effort would be lower for decision makers who select from similar ideas in subsets compared to decision makers who select from random ideas. The univariate test statistics show significant differences for consideration set size (F (1, $81)=6.146, p=0.015$, partial $\left.\eta^{2}=0.071\right)$ but not for perceived cognitive effort $(\mathrm{F}(1,81)=1.389, \mathrm{p}>0.05$, partial $\left.\eta^{2}=0.017\right)$. Decision makers who chose or dropped ideas from subsets with similar ideas had on average 45.65 out of 75 ideas in their final consideration sets compared to 51.24 out of 75 ideas for decision makers presented with subsets of randomly allocated ideas. Hence, H3 is supported. Moreover, the cognitive effort was perceived lower in the similarity treatment $(\mathrm{M}=3.28)$ than in the random treatment $(\mathrm{M}=3.37)$. This direction was as expected, but the difference was not significant and therefore $\mathrm{H} 4$ is not supported.

\section{Controls}

We further investigated the significant multivariate main effects of the control variables (see Table 2).

There exists a significant univariate main effect of gender on consideration set size $(\mathrm{F}(1,81)=8.604, \mathrm{p}=$ $0.004)$. Men selected more ideas $(\mathrm{M}=52.61, \mathrm{SD}=12.75)$ than women $(\mathrm{M}=44.02, \mathrm{SD}=16.03)$. There was no significant main effect of gender on perceived cognitive effort $(F(1,81)=0.000, p>0.05)$.

We found a significant univariate main effect for need for cognition on consideration set size $(F(1,81)=$ $5.978, \mathrm{p}=0.017)$. Yet, further bi-variate correlation analysis revealed no significant direct association between need for cognition and consideration set size. We also found a significant univariate main effect for perceived need for cognition on cognitive effort $(\mathrm{F}(1$, $81)=6.066, p=0.016)$. Individuals with low need for cognition perceived the task to be more cognitively effortful $(\mathrm{M}=3.58, \mathrm{SD}=0.71)$ than individuals with high need for cognition $(\mathrm{M}=3.14, \mathrm{SD}=0.64)$.

Table 2: Results of hypotheses tests

\begin{tabular}{|c|c|c|c|c|c|}
\hline Source & $\mathrm{DF}$ & $\begin{array}{l}\text { Mean } \\
\text { square }\end{array}$ & $\mathrm{F}$ & p-value & Partial $\eta 2$ \\
\hline \multicolumn{6}{|c|}{ ANOVA Dependent variable: Consideration set size } \\
\hline Selection strategy & 1 & 12004.933 & 208.825 & 0.000 & 0.721 \\
\hline Idea similarity & 1 & 353.318 & 6.146 & 0.015 & 0.071 \\
\hline Gender & 1 & 494.609 & 8.604 & 0.004 & 0.096 \\
\hline Domain expertise & 1 & 230.633 & 4.012 & 0.049 & 0.047 \\
\hline Need for cognition & 1 & 343.691 & 5.978 & 0.017 & 0.069 \\
\hline Error & 81 & 57.488 & & & \\
\hline \multicolumn{6}{|c|}{ ANOVA Dependent variable: Perceived cognitive effort } \\
\hline Selection strategy & 1 & 2.310 & 5.268 & 0.024 & 0.061 \\
\hline Idea similarity & 1 & 0.609 & 1.389 & 0.242 & 0.017 \\
\hline Gender & 1 & 0.000 & 0.000 & 0.997 & 0.000 \\
\hline Domain expertise & 1 & 0.255 & 0.581 & 0.448 & 0.255 \\
\hline Need for cognition & 1 & 2.660 & 6.066 & 0.016 & 2.660 \\
\hline Error & 81 & 0.438 & & & \\
\hline
\end{tabular}




\section{Implications, Limitations and Future Research}

This research set out to investigate which digital nudges can achieve improved convergence outcomes in open innovation initiatives. We tested the digital nudges "selection strategy" and "idea subset similarity" and found that "selection strategy" is significantly associated with consideration set size and perceived cognitive effort while "idea subset similarity" is also significantly associated with consideration set size but only weakly significantly associated with perceived cognitive effort.

Theoretical implications. We provide empirical evidence that the digital nudge of "selection strategy" successfully prompted individuals towards either including (choose) promising or excluding (drop) nonpromising ideas. Our findings show that individuals prompted with an inclusion strategy form smaller consideration sets than under an exclusion strategy. This confirms the effects of inclusion/exclusion on consideration set size in a setting of convergence with crowd-generated ideas.

In addition, individuals prompted with inclusion also showed higher perceived cognitive effort. This finding suggests that in the inclusion treatment participants adapted decision strategies that process alternatives in depth and hence require more cognitive resources. Hence, our findings contribute an additional relationship between inclusion/exclusion strategies and perceived cognitive effort to theory.

Moreover, this study provides empirical evidence that there exists a relationship between the digital nudge "idea subset similarity" and consideration set size. Our findings show that decision makers created significantly smaller consideration sets when the convergence system presented them with subsets defined by idea similarity. To our surprise, we did not find empirical evidence that "idea subset similarity" was significantly associated with perceived cognitive effort. The direction of our theorizing is supported but the difference was not significant on the 0.05 level. A reason could be that the subset size of five as an intervention already sufficiently decreased cognitive effort to curb choice deferral [44]. Hence, our findings add a new hypothesis to the body of literature on selection that consideration set sizes are smaller when people can choose from choice sets that include similar alternatives rather than from randomly arranged choice sets.

Practical implications. Our findings have also implications for managers and designers. Managers of open innovation initiatives can benefit from our findings in two ways. First, if their concern is to drastically reduce a huge number of ideas into a shortlist, they should prompt their raters towards an inclusion strategy and present them with subsets of similar ideas. However, the inclusion strategy might demand quite substantial perceived cognitive effort from the raters. Second, if the concern is to reduce perceived cognitive effort, they should prompt their raters towards exclusion. However, in this case managers need to anticipate larger consideration sets. This is an interesting trade-off. Either opt for larger consideration sets and lower perceived cognitive effort or smaller consideration sets and higher perceived cognitive efforts. It suggests that managers should get to know their crowd workers whether they enjoy effortful thinking tasks or not. If this is the case, managers can achieve the best convergence outcomes by adopting the "idea strategy" digital nudge of exclusion.

Limitations. There also exist some limitations that need to be considered when drawing on our findings. Our idea similarity manipulation needs to be interpreted carefully because subjects did not perceive subsets as significantly more similar in this treatment condition than in the random condition. This perception might have been influenced by the decreasing level of similarity among ideas the further decision makers proceeded in the assessment. Such decreasing similarity is a function of the algorithm we employed for decomposition of ideas into subsets.

Moreover, we drew on a small student population to mimic a crowd of non-experts. It could be that the effects of idea similarity change once the assessment is performed by individuals with high domain expertise. Further data collection is necessary to increase sample size.

Future research. The reported research discovered novel relevant relationships between digital nudges and convergence outcomes. The underlying behavioral and cognitive mechanisms, which explain these relationships were theorized but not tested. Hence, future research should dig deeper into the discovered relationships and set out to find empirical evidence that supports (or fails to support) these relationships.

While this study compares inclusion to exclusion strategies in convergence of crowd-generated ideas, future research could investigate how effects change when the digital nudge "selection strategy" is less restrictive and allows to choose and drop ideas at the 
same time or to apply a sequence of inclusion and exclusion in a multi-stage convergence process.

Future research could also investigate if the digital nudge idea subset similarity is more influential at the beginning or the end of a decision making process. Future research could assess the effectiveness of this nudge of idea similarity by comparing the results of the first rounds where the perception of similarity among ideas was likely to be high with later rounds of less similar ideas. Moreover, it would be interesting to find out if the effectiveness of idea subset similarity depends on sizes of subsets. While our algorithm used a fixed subset size of five ideas, future research might explore the effects of an endogenous definition of subset size based on ideas similarity or compare small versus large subsets.

In this study, participants had to decide on the most promising ideas from 75 ideas. While the number of ideas presented appears to be big, each of the ideas were described in one sentence. It is likely that perceived cognitive effort would increase considerably with an increase of text length and potentially more inherent features. Hence, future research could replicate this study but switch to ideas with longer descriptions.

\section{Acknowledgements}

The research leading to the presented results was partially funded by the Austrian Science Fund (FWF): P 29765.

\section{References}

[1] W. Chesbrough, Open Innovation: The New Imperative for Creating and Profiting from Technology. 2006.

[2] A. King and K. R. Lakhani, "Using Open Innovation to Identify the Best Ideas," MIT Sloan Manag. Rev., vol. 55, no. 1, pp. 41-48, 2013.

[3] K. Girotra, C. Terwiesch, and K. T. Ulrich, "Idea Generation and the Quality of the Best Idea,” Manage. Sci., vol. 56, no. 4, pp. 591-605, 2010.

[4] G. Jouret, “Inside Cisco's search for the next big idea," Harv. Bus. Rev., vol. September, pp. 1-7, 2009.

[5] O. M. Bjelland and R. C. Wood, "An Inside View of IBM' $s$ ' Innovation Jam ' An Inside View of IBM' s ' Innovation Jam," MIT Sloan Manag. Rev., vol. 50, no. 1, pp. 32-40, 2008.

[6] A. Sukhov, "The role of perceived comprehension in idea evaluation," Creat. Innov. Manag., no. January, pp. 183-195, 2018.
[7] B. Onarheim and B. T. Christensen, "Distributed idea screening in stage-gate development processes," J. Eng. Des., vol. 23, no. 9, pp. 660-673, 2012.

[8] C. Faure, "Beyond brainstorming: Effects of different group procedures on selection of ideas and satisfaction with the process," J. Creat. Behav., vol. 38, no. 1, pp. 13-34, 2004.

[9] L. J. Kornish and J. Hutchison-Krupat, "Research on Idea Generation and Selection: Implications for Management of Technology," Prod. Oper. Manag., vol. 26, no. 4, pp. 633$651,2017$.

[10] I. Blohm, C. Riedl, J. Füller, and J. M. Leimeister, "Rate or Trade? Identifying Winning Ideas in Open Idea Sourcing," Inf. Syst. Res., no. April, pp. 1-53, 2016.

[11] I. Seeber, R. Maier, G.-J. de Vreede, and B. Weber, "Beyond Brainstorming: Exploring Convergence in Teams," J. Manag. Inf. Syst., vol. 17, no. 4, pp. 1-42, 2017.

[12] P. G. Hansen, "The definition of nudge and libertarian paternalism: Does the hand fit the glove?," Eur. J. Risk Regul., vol. 7, no. 1, pp. 155-174, 2016.

[13] M. Weinmann, C. Schneider, and J. vom Brocke, "Digital Nudging," Bus. Inf. Syst. Eng., vol. 58, no. 6, pp. 433-436, 2016.

[14] R. H. Thaler and C. R. Sunstein, Nudge: Improving Decisions About Health, Wealth, and Happiness. New Haven: Yale University Press, 2009.

[15] C. R. Sunstein, "Nudging: A Very Short Guide," J. Consum. Policy, vol. 37, no. 4, pp. 583-588, 2014.

[16] S. Stieglitz, T. Potthoff, and T. Kißmer, "Digital Nudging am Arbeitsplatz," HMD Prax. der Wirtschaftsinformatik, pp. 965-976, 2017.

[17] D. Kahneman, Thinking Fast and Slow. Macmillan, 2011.

[18] J. S. B. T. Evans, "Dual-Processing Accounts of Reasoning, Judgment, and Social Cognition," Annu. Rev. Psychol., vol. 59, no. 1, pp. 255-278, 2008.

[19] E. J. Johnson and J. W. Payne, "Effort and Accuracy in Choice," Manage. Sci., vol. 31, no. 4, pp. 395-414, 1985.

[20] Daniel Kahneman, Amos Tversky, and Paul Slovic, Judgment under Uncertainty: Heuristics \& Biases, vol. 16, no. 185. 1974.

[21] M. Klein and A. C. B. Garcia, "High-speed idea 
filtering with the bag of lemons," Decis. Support Syst., vol. 78, pp. 39-50, 2015.

[22] D. Heller, I. P. Levin, and M. Goransson, "Selection of strategies for narrowing choice options: Antecedents and consequences," Organ. Behav. Hum. Decis. Process., vol. 89, no. 2, pp. 1194-1213, 2002.

[23] I. Levin, M. Huneke, and J. Jasper, "Information Processing at Successive Stages of Decision Making: Need for Cognition and InclusionExclusion Effects.," Organ. Behav. Hum. Decis. Process., vol. 82, no. 2, pp. 171-193, 2000.

[24] E. F. Rietzschel, B. a Nijstad, and W. Stroebe, "The selection of creative ideas after individual idea generation: choosing between creativity and impact.," Br. J. Psychol., vol. 101, no. Pt 1, pp. 47-68, Feb. 2010.

[25] I. Seeber, A. B. Merz, R. Maier, G.-J. de Vreede, and B. Weber, "Convergence on SelfGenerated vs. Crowdsourced Ideas in Crisis Response: Comparing Social Exchange Processes and Satisfaction with Process," Proc. 50th Hawaii Int. Conf. Syst. Sci., pp. 1-10, 2017.

[26] P. M. Di Gangi, M. M. Wasko, and R. E. Hooker, 'Getting Customers' Ideas to Work for You: Kearning from Dell how to Succeed with Online User Innovation Communities," MIS Q. Exec., vol. 9, no. 4, pp. 197-212, 2010.

[27] T. P. Walter and A. Back, "A text mining approach to evaluate submissions to crowdsourcing contests," Proc. Annu. Hawaii Int. Conf. Syst. Sci., pp. 3109-3118, 2013.

[28] L. J. Kornish and K. T. Ulrich, "Opportunity Spaces in Innovation: Empirical Analysis of Large Samples of Ideas," Manage. Sci., vol. 57, no. 1, pp. 107-128, 2011.

[29] O. Toubia and O. Netzer, "Idea Generation , Creativity, and Prototypicality," Mark. Sci., vol. 25, no. 5, pp. 411-425, 2016.

[30] B. Scheibehenne, R. Greifeneder, and P. M. Todd, "Can There Ever Be Too Many Options? A Meta-Analytic Review of Choice Overload," J. Consum. Res., vol. 37, no. 3, pp. 409-425, 2010.

[31] H. A. Simon, “A Behavioral Model Of Rational Choice," Q. J. Econ., vol. 69, no. 1, pp. 99-118, 1955.

[32] A. Fox, C., \& Tversky, "Ambiguity Aversion and Comparative Ignorance," Q. J. Econ., vol. 110, no. 3, pp. 585-603, 1995.

[33] J. G. Griffin and S. M. Broniarczyk, "The
Slippery Slope: The Impact of Feature Alignability on Search and Satisfaction," $J$. Mark. Res., vol. 47, no. 2, pp. 323-334, 2010.

[34] A. Chernev, U. Böckenholt, and J. Goodman, "Choice overload: A conceptual review and meta-analysis," J. Consum. Psychol., vol. 25, no. 2, pp. 333-358, 2012.

[35] D. Ramage, A. N. Rafferty, and C. D. Manning, "Random Walks for Text Semantic Similarity," in Proceedings of the 2009 Workshop on Graph-based Methods for Natural Language Processing, 2009, pp. 23-31.

[36] A. Kashyap et al., Robust semantic text similarity using LSA, machine learning, and linguistic resources, vol. 50, no. 1. Springer Netherlands, 2016.

[37] J. Ross, A. Zaldivar, L. Irani, and B. Tomlinson, "Who are the Turkers? Worker Demographics in Amazon Mechanical Turk," Chi Ea 2010, no. July 2016, pp. 2863-2872, 2010.

[38] A. Westbrook and T. S. Braver, "Cognitive effort: A neuroeconomic approach," Cogn. Affect. Behav. Neurosci., vol. 15, no. 2, pp. 395415, 2015.

[39] R. Pereira, "Influence of query-based decision aids on consumer decision making in electronic commerce," Inf. Resour. Manag. J., vol. 14, no. 1, pp. 31-48, 2001.

[40] W. Wang and I. Benbasat, "Interactive Decision Aids For Consumer Decision Making in ECommerce: The Influence of Perceived Strategy Restrictiveness," MIS Q., vol. 33, no. 2, pp. 293-320, 2009.

[41] K. Y. Tam and Y. H. Schuk, "Web Personalization as a Persuasion Strategy: An Elaboration Likelihood Model Perspective," Inf. Syst. Res., vol. 16, no. 3, pp. 271-291, 2005.

[42] J. C. Nunnally and I. H. Bernstein, "Psychometric theory," 1978.

[43] J. F. Hair, W. C. Black, B. J. Babin, and R. E. Anderson, Multivariate data analysis: a global perspective. Pearson Education, 2010.

[44] L. E. Pilli and J. A. Mazzon, "Information overload, choice deferral, and moderating role of need for cognition: Empirical evidence," Rev. Adm., vol. 51, no. 1, pp. 036-055, 2016. 